\title{
Reconfigurable Sampling of the Electric Field at the Reflecting Surface of Folded Arrayed Waveguide Grating Multiplexers
}

\author{
A. A. Bernussi, L. Grave de Peralta, M. Knapczyk, R. Gale, and H. Temkin
}

\begin{abstract}
We describe the use of a reflective arrayed waveguide grating multiplexer in conjunction with a digital micromirror device to modulate the electric field distribution in the grating. Modulation of the electric field is accomplished by turning individual digital mirrors ON and OFF. By imposing a reconfigurable grating superstructure on the digital mirror array, we can change the free-spectral range of the device and alter the overall transmission spectra. The experimental results are in excellent agreement with model calculations. This type of a device is expected to be useful in generation of reconfigurable sequences of ultrashort pulses.
\end{abstract}

Index Terms-Amplitude modulation, arrayed waveguide grating (AWG), digital micromirrors, electric field, multiplexer, pulse generation, reconfigurable grating.

$\mathbf{S}$ ILICA-BASED arrayed waveguide gratings (AWGs) have been developed for applications in optical networks based on wavelength-division multiplexing (WDM) [1]-[3]. Recently, the use of AWGs as pulse shapers in ultrafast pulse generation has received considerable attention [4]-[6]. Although different approaches to AWGs have been successfully demonstrated, these devices continue to be passive.

In this work, we present experiments modifying the output response of AWGs. Our approach combines a reflective (R)-AWG [7] with an array of digital micromirrors, known as a digital mirror device (DMD), used as an external reflector. This allows us to periodically sample the grating of the R-AWG to modify its overall output response. The output spectra are dynamically reconfigured by digital amplitude modulation of the electric field of the waveguide grating. We demonstrate that the free-spectral range (FSR) of an R-AWG can be modified by controlling the ON-OFF state of groups of micromirrors. The possibility of active modification of the FSR has potential applications in the generation of ultrafast pulses with high-repetition rates for hybrid WDM and optical time-division multiplexing networking [4].

Performance characteristics of R-AWGs used in this work were described previously [7]. Gaussian response devices were designed for 40-channel operation with the channel-to-channel separation of $100 \mathrm{GHz}$. Silica-based R-AWGs were fabricated in a conventional silicon-integrated circuit foundry using $\mathrm{SiO}_{2}$

Manuscript received May 12, 2004; revised June 7, 2004. This work was supported by Texas Instruments and by the Jack F. Maddox Foundation.

The authors are with the Department of Electrical and Computer Engineering, Texas Tech University, Lubbock, TX 79409 USA (e-mail: ayrton.bernussi@coe.ttu.edu).

Digital Object Identifier 10.1109/LPT.2004.834494 layers deposited on wafers of silicon. The relative refractive index difference between the core and cladding materials of the waveguide was $\Delta=0.68 \%$. High reflectivity of the reflecting surface folding the grating was assured by deposition of a $\mathrm{Cr}-\mathrm{Au}$ layer. In the experiments discussed here, the $\mathrm{Cr}-\mathrm{Au}$ layer was removed and an external DMD was used as a reflector instead.

A DMD array of $1024 \times 768$ elements was used as the external reflector [8]. Individual aluminum micromirrors were 13 $\times 13 \mu \mathrm{m}$ in size. Depending on the control signal applied to the underlying memory cell, each mirror rotates until its tip touches the landing electrode. An ON signal rotates the mirror by $+12^{\circ}$, while an OFF signal causes rotation of $-12^{\circ}$ with respect to the unpowered mirror plane [8]. The DMD device was positioned at a $+12^{\circ}$ angle with respect to the grating waveguides of the AWG. As a result, the reflecting surfaces of the DMD micromirrors in the ON state were perpendicular to the grating waveguides. In this configuration, the light coupled into the waveguides of the grating is reflected back and contributes to the output response of the AWG. When the DMD is set to the OFF state, light is not coupled back to the grating. Waveguides of the R-AWG grating can be sampled by controlling the ON-OFF state of each micromirror. This is accomplished in a reconfigurable fashion and allowing for control of the transmission response of the R-AWG on a time scale of microseconds.

In order to place the external micromirrors closely $(\sim 10 \mu \mathrm{m})$ to the reflecting surface of the grating of the R-AWG the cover glass of the DMD package was removed. In addition, in order to suppress reflections at the silica waveguides-air interface, the reflecting surface of the R-AWG grating was polished at an $8^{\circ}$ angle. The R-AWGs were designed with a constant distance of $\sim 31 \mu \mathrm{m}$ between consecutive waveguides of the grating, while the distance between consecutive mirrors in the DMD is about $14 \mu \mathrm{m}$. The pitch mismatch results in intensity errors and an additional loss penalty of $2 \mathrm{~dB}$, that contributes to the output response of the R-AWG.

The output spectrum of the R-AWG strongly depends on the number of grating waveguides contributing $\left(N_{\mathrm{wg}}^{\mathrm{ON}}\right)$ and not contributing $\left(N_{\mathrm{wg}}^{\mathrm{OFF}}\right)$ to the device response. When the grating waveguides are sampled periodically by the micromirrors of the DMD, a coarse waveguide grating is formed with a period $\left(d_{B}\right)$ expressed as $d_{B}=\left[\left(N_{\mathrm{wg}}^{\mathrm{ON}}-1\right)+\left(N_{\mathrm{wg}}^{\mathrm{OFF}}+1\right)\right] d$, where $d$ is the distance between waveguides at the grating-slab interface. Examples of a single channel output response of an active R-AWG with the waveguide grating sampled with different periods are shown in Fig. 1. Similar results were obtained for other output channels of the device. When all the micromirrors 


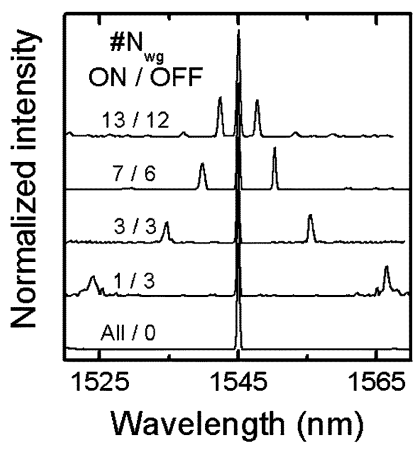

Fig. 1. Output response of a single channel of an R-AWG with grating waveguides sampled with different periods. Intensities of individual spectra were normalized in order to make direct comparisons.

of the DMD are set to the ON-state, the single-channel output response of the $C$-band R-AWG consisted of a peak centered at $\sim 1545 \mathrm{~nm}$. Measurements of the R-AWG response of the same output channel, but sampling the grating with different periods, revealed the presence of multiple transmission peaks. The main peak remains centered at the same position as that obtained with all the micromirrors set to the ON state. However, its intensity was reduced with increased $N_{\mathrm{wg}}^{\mathrm{OFF}}$. Additional peaks were observed symmetrically positioned around the main peak. The origin of these peaks can be understood by analyzing the equations relating the center wavelength $\left(\lambda_{o}\right)$, the diffraction order $(m)$, and the wavelength separation $(\Delta \lambda)$ between the central peak and the next order peak as a function of the R-AWG design parameters [7], [9]

$$
\begin{aligned}
\lambda_{o} & =2 \frac{n_{c}}{m} \Delta L \\
m & =\frac{n_{s} D N_{c}}{f \Delta \lambda_{\mathrm{ch}} N_{g}} d \\
\Delta \lambda & = \pm \frac{\lambda_{o} f \Delta \lambda_{\mathrm{ch}}}{D n_{s}} \frac{1}{d_{B}}
\end{aligned}
$$

where in (3) the standard distance between waveguides at the grating-slab interface $d$ is replaced with $d_{B}$. The $n_{c} \Delta L$ is the optical path-length difference between waveguides of the grating, $n_{s}$ is the effective refractive index of the slab region, $D$ is the distance between waveguides at the coupler-slab interface, $f$ is the radius of curvature of the slab waveguide, $\Delta \lambda_{\mathrm{ch}}$ is the wavelength separation between consecutive output channels, $N_{c}$ and $N_{g}$ are the group refractive index of the grating waveguides and the index of the material filling the gap between the external mirror and grating reflecting surface, respectively. Periodic sampling of the grating waveguides at the reflecting surface of the R-AWG results in $d$ and $\Delta L$ being multiplied by a common factor of $\left(N_{\mathrm{wg}}^{\mathrm{OFF}}+1\right)$. Substituting the modified values of $d$ and $\Delta L$ in (1) and (2) results in no alteration of the wavelength of the output channel. This is consistent with the data of Fig. 1, where the main peak position of the output channel spectra of R-AWG sampled with different periods are identical to the one obtained with the all micromirrors set in the ON-state. The additional peaks observed in the spectra are attributed to higher (lower) diffraction orders of the active R-AWG. The slight asymmetry observed in the
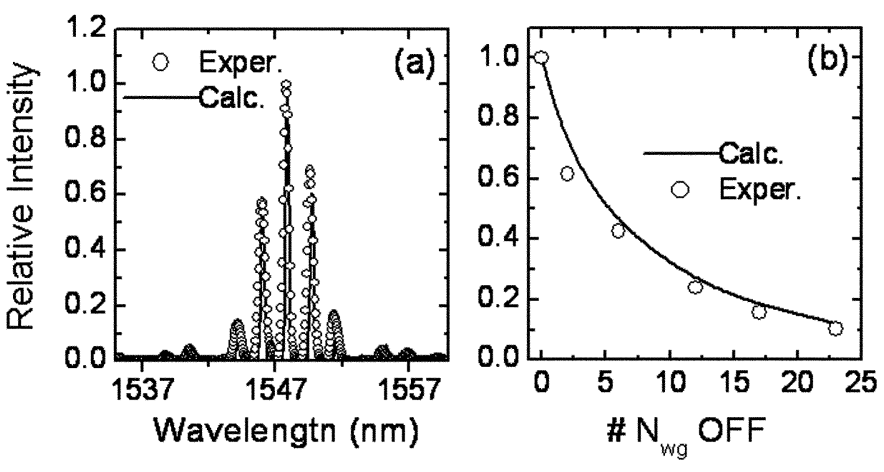

Fig. 2. Simulated and measured single channel (a) output spectrum response with grating waveguides sampled with $N_{\mathrm{wg}}^{\mathrm{ON}}=13, N_{\mathrm{wg}}^{\mathrm{OFF}}=23$ and (b) relative channel peak intensity of the fundamental order emission for grating waveguides sampled with $N_{\mathrm{wg}}^{\mathrm{ON}}=13$ and different values of $N_{\mathrm{wg}}^{\mathrm{OFF}}$.

intensity of the additional peaks is attributed to the pitch mismatch between the micromirrors and the grating waveguides. Equation (3) shows that the wavelength separation $\Delta \lambda$ between the fundamental and the adjacent order peaks decreases as $d_{B}$ increases. This is also consistent with the data shown in Fig. 1, where $\Delta \lambda$ decreases as the modulation period $N_{\mathrm{wg}}^{\mathrm{ON}}+N_{\mathrm{wg}}^{\mathrm{OFF}}$ increases. As an example, in the case of a sampled grating with $N_{\mathrm{wg}}^{\mathrm{ON}}=13, N_{\mathrm{wg}}^{\mathrm{OFF}}=12$ we determine, from the data of Fig. $1, \Delta \lambda=+2.64$ and $-2.66 \mathrm{~nm}$. Using the device design parameters [7] and (3), we calculate $\Delta \lambda= \pm 2.64 \mathrm{~nm}$, in excellent agreement with experimental results. This clearly demonstrates that the FSR of the active R-AWG can be systematically modified by digital modulation of the electric field at the reflecting surface folding the grating.

In order to analyze the effect of the electric field amplitude modulation of the waveguide grating on the transmission characteristics of R-AWGs, we simulated the device response using the AWG field model in the single Gaussian approximation [10]. Using the device design parameters defined above and selecting only those grating waveguides $\left(N_{\mathrm{wg}}^{\mathrm{ON}}\right)$ contributing to the response of the device, we calculated the single-channel transmission spectra for different values of $N_{\mathrm{wg}}^{\mathrm{OFF}}$. A comparison between simulated and measured single channel output spectra of an R-AWG with grating waveguides sampled with $N_{\mathrm{wg}}^{\mathrm{ON}}=$ $13, N_{\mathrm{wg}}^{\mathrm{OFF}}=23$ is shown in Fig. 2(a). Higher diffraction orders, rather than only the adjacent ones, are clearly observed in both simulated and experimental spectra. An excellent agreement between theory and experiment is evident.

As mentioned above, the overall intensity of the output response of the R-AWGs with sampled waveguide grating decreases with increased values of $N_{\mathrm{wg}}^{\mathrm{OFF}}$. A comparison between simulated and measured single channel peak intensity of the fundamental order transmission, normalized to the main peak intensity corresponding to all micromirrors being in the ON-state, for $N_{\mathrm{wg}}^{\mathrm{ON}}=13$ and different values of $N_{\mathrm{wg}}^{\mathrm{OFF}}$ is shown in Fig. 2(b). The measured relative intensity decreases exponentially with $N_{\mathrm{wg}}^{\mathrm{OFF}}$ in good agreement with simulation results.

As shown above [Figs. 1 and 2(a)], the transmission wavelengths and the relative intensity of higher (lower) order peaks of sampled grating R-AWGs depend on the modulation period. Fig. 3 shows normalized single-channel spectra of an R-AWG 


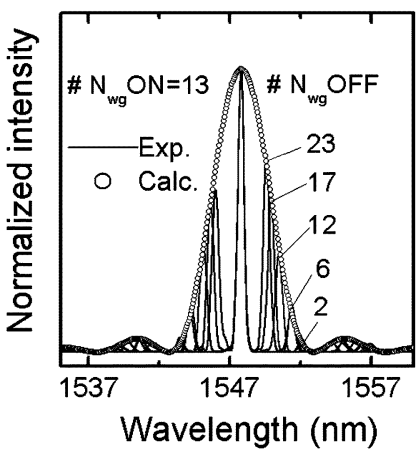

Fig. 3. Single-channel output response measured for an R-AWG with grating waveguides sampled with $N_{\mathrm{wg}}^{\mathrm{ON}}=13$ and different values of $N_{\mathrm{wg}}^{\mathrm{OFF}}$. Circles represent the calculated envelope spectrum.

with the waveguide grating sampled with $N_{\mathrm{wg}}^{\mathrm{ON}}=13$ but with different values of $N_{\mathrm{wg}}^{\mathrm{OFF}}$. As $N_{\mathrm{wg}}^{\mathrm{OFF}}$ increases, the relative intensity of the higher order peaks increases and their wavelength shifts systematically closer to the main peak. This results in a well-defined spectral envelope distribution for higher (lower) diffraction order peaks. This envelope can be modeled by performing a Fourier transform of the grating section $\left(N_{\mathrm{wg}}^{\mathrm{ON}}\right)$ that contributes to the response of the R-AWG. The sampled waveguide grating has a rectangular spatial profile. This gives rise to an output response $(I)$ that can be described by a sinc envelope function [10]

$$
I(\delta \lambda) \propto\left[\frac{\sin (k \delta \lambda)}{(k \delta \lambda)}\right]^{2}
$$

where $k=\left(\pi m L_{B} / \lambda_{o} d\right)$ and $\delta \lambda$ is the wavelength shift. The calculated envelope spectrum of the R-AWG using (4), $N_{\mathrm{wg}}^{\mathrm{ON}}=$ 13 and the design parameters of the R-AWG discussed previously [7], is also shown in Fig. 3. Good agreement with the measurement is again very clear.

We believe the unique properties of active R-AWGs will have many novel applications. One area of interest is in control of high-speed pulses [11]-[13], where AWG- type devices are used for pulse shaping [5], [6]. The active device described here will have a number of advantages over passive pulse shapers. The separation between pulses within a high-speed pulse train is inversely proportional to the FSR of the AWG [5]. By changing the FSR with an external array of micromirrors, the separation between pulses can be changed. In addition, by changing the $N_{\text {wg }}^{\mathrm{ON}}$ in the grating superstructure formed by the DMD, the width of individual output pulses could be adjusted.

In a conventional AWG, interference occurs because of temporal overlap of light sampling different waveguides of the grating. Once the pulsewidth becomes shorter than the propagation time delays between different groups of waveguides, this temporal overlap disappears. As a result, the output of the AWG consists of a high-speed pulse train [5]. In this case, if $N_{\text {wg }}^{\mathrm{ON}}=1$, each waveguide contributes one pulse to the output train and the width of each pulse is equal to that of the input source [5]. On the other hand, when $N_{\mathrm{wg}}^{\mathrm{ON}}>1$ and $N_{\mathrm{wg}}^{\mathrm{OFF}}$ is sufficiently large, each group of waveguides contributes a pulse to the output train with the pulsewidth increased by a factor proportional to $N_{\mathrm{wg}}^{\mathrm{ON}}$. In the DMD-AWG combination described here, the pulsewidth becomes adjustable. The number of pulses in the pulse train is equal to the number of ON-state groups of mirrors. By changing the state of individual groups, a given pulse will be removed from the train. This gives rise to the possibility of forming a unique sequence of pulses for each DMD configuration. A full implementation of this idea requires a match between the waveguide period of the AWG and the mirror period of the DMD.

In summary, we demonstrate an active R-AWG obtained with an array of digital-mirrors used as the external reflector. Control of the micromirror state enables amplitude modulation of the electric field of light captured by grating waveguides. The electric field modulation allows dynamic modification of the FSR. The observation of additional transmission peaks in the same channel output response, symmetrically located at both lower and higher wavelength from the main peak, confirms dynamic control of the FSR. The observed output response of active R-AWGs was found to be in excellent agreement with simulation results.

\section{REFERENCES}

[1] Y. Hibino, "Recent advances in high-density and large-scale AWG multi/demultiplexers with higher index-contrast silica-based PLCs," IEEE J. Select. Topics Quantum Electron., vol. 8, pp. 1090-1101, Nov./Dec. 2002.

[2] C. Dragone, "An $N \times N$ optical multiplexer using a planar arangement of two star couplers," IEEE Photon. Technol. Lett., vol. 3, pp. 812-815, Sept. 1991

[3] M. K. Smit, "New focusing and dispersive planar component based on an optical phased array," Electron. Lett., vol. 24, pp. 385-386, 1988.

[4] I. Y. Khrushchev, J. D. Bainbridge, J. E. A. Whiteaway, I. H. White, and R. V. Penty, "Multiwavelength pulse source for OTDM/WDM applications based on arrayed waveguide grating," IEEE Photon. Technol. Lett., vol. 11, pp. 1659-1671, Dec. 1999.

[5] D. E. Leaird, A. M. Weiner, S. Shen, A. Sugita, S. Kamei, M. Ishii, and K. Okamoto, "Generation of high repetition rate WDM pulse trains from an arrayed-waveguide grating," IEEE Photon. Technol. Lett., vol. 13, pp. 221-223, Mar. 2001

[6] D. E. Leaird, A. M. Weiner, S. Kamei, M. Ishii, A. Sugita, and K. Okamoto, "Double-passed arrayed waveguide grating for $500-\mathrm{GHz}$ pulse burst generation," IEEE Photon. Technol. Lett., vol. 14, pp. 1451-1453, Oct. 2002.

[7] L. G. de Peralta, A. A. Bernussi, S. Frisbie, R. Gale, and H. Temkin, "Reflective arrayed waveguide grating multiplexer," IEEE Photon. Technol. Lett., vol. 15, pp. 1398-1400, Oct. 2003.

[8] M. A. Magnardi, "From IC's to DMD's," TI Tech. J., vol. 15, pp. 56-63, 1998.

[9] K. Okamoto and H. Yamada, "Arrayed-waveguide grating multiplexer with flat spectral response," Opt. Lett., vol. 20, pp. 43-45, 1995.

[10] P. Muñoz, D. Pastor, and J. Capmany, "Modeling and design of arrayed waveguide gratings," J. Lightwave Technol., vol. 20, pp. 661-674, Apr. 2002.

[11] C. Froehly, B. Colombeau, and M. Vampouille, Progress in Optics, E. Wolf, Ed. North-Holland, Amsterdam, 1983, vol. 10, pp. 115-121.

[12] D. E. Leaird and A. M. Weiner, "Femtosecond optical packet generation by a direct space-to-time pulse shaper," Opt. Lett., vol. 24, pp. 853-855, 1999.

[13] K. Takiguchi, T. Kominato, H. Takahashi, T. Shibata, and K. Okamoto, "Flexible pulse waveform generation using a silica waveguide based spectrum synthesis circuit," in Tech. Dig. Opitcal Fiber Communication (OFC) Conf., Los Angeles, CA, Feb. 22-27, 2004. 